\title{
Dynamic Modelling and Trajectory Tracking Control of Unmanned Tracked Vehicles
}

\author{
Ting Zou ${ }^{\mathrm{a}, *}$, Jorge Angeles ${ }^{\mathrm{b}}$, Ferri Hassani $^{\mathrm{c}}$ \\ ${ }^{a}$ Department of Mechanical Engineering, Memorial University of Newfoundland, St. John's, \\ NL, Canada \\ ${ }^{b}$ Centre for Intelligent Machines, Department of Mechanical Engineering, McGill \\ University, Montréal, QC, Canada \\ ${ }^{c}$ Department of Mining and Materials Engineering, McGill University, Montréal, QC, \\ Canada
}

\begin{abstract}
Tracked vehicles have inherent advantages over wheeled vehicles, as the former provide stable locomotion on loose and uneven terrain. However, compared with the latter, the slippage generated due to the complex, nonlinear track-terrain interactions during skid-steering to follow a curve, brings about difficulties preventing the accurate prediction of their motions. The key to improving the accuracy of trajectory-following is the "proper" motion control methodology that can accurately factor-in the slippage behavior. In this paper, the authors propose a novel approach to the dynamic modeling and motion control of tracked vehicles undergoing skid-steering on horizontal, hard terrain, under nonholonomic constraints. Due to the skew-symmetry property of nonholonomic mechanical systems, the control methodology is established using the backstepping method based on a modified Proportional-Integral-Derivative (PID) computed-torque control. A key element in the control strategy proposed here is the reliable estimation of the pose - position and orientation - of the vehicle platform and its twist - point velocity and angular velocity. It is assumed that the vehicle is suitably instrumented to allow for accurate-enough pose and twist estimates. Validated via a numerical example, the proposed controller is proven to be ef-
\end{abstract}

\footnotetext{
* Corresponding author

Email addresses: ting.zou@mail.mcgill.ca (Ting Zou), angeles@cim.mcgill.ca (Jorge Angeles), ferri.hassani@mcgill.ca (Ferri Hassani)
} 
fective in controlling an unmanned tracked vehicle.

Keywords: tracked vehicle, skew-symmetry property, trajectory tracking control, planar kinematics, nonholonomic constraint

\section{Introduction}

The motion control of nonholonomic mechanical systems has attracted considerable research interest in recent years. Such systems have found extensive applications in industry, including wheeled mobile robots, wheeled vehicles, tracked vehicles, etc. Compared with wheeled vehicles, tracked vehicles are widely used in applications that require high mobility over rough terrain, due to the high traction they provide. This feature brings significant advantages over their wheeled counterparts in various application domains, such as agriculture, nuclear sites, and so on. Tracked mining vehicles in open-pit mining, a.k.a. blasthole drilling, are typical application examples. Equipped with two parallel actuated tracks, the rig carrying a drill mast with proper equipment, drills narrow holes vertically deep into the ground, which are filled with explosives afterwards [1]. The hazardous working environment of the drilling process calls for minimizing exposure of the human operator to the risk, which is also a technical challenge [2]. Autonomous tracked vehicles have experienced fast development during the past years to address this challenge [3]. Developing semi-autonomous or fully autonomous blasthole-drill is a major research objective in the mining industry. Instead of requiring a human operator on the vehicle, autonomous blasthole-drill is supervised by an operator from a remote location, using stateof-the-art communications and control technology. This approach should lead to safe, efficient and smooth drilling operations. Precision and operating efficiency are further improved by the realization of the remote control of multiple blasthole drills. Under these conditions, a remote operator can use one single console to coordinate and control multiple blasthole drill rigs, thus significantly simplifying the working process of one-to-one control, i.e., one remote operator controlling one single drill rig, thereby maximizing productivity. 
The motion control of tracked vehicles is a complex problem, related to various types of maneuverability, involving straight motion, high-speed turning, skid-steering, etc. The steering of tracked vehicles is unique, making it different from their wheeled counterparts. Several types of steering are involved, including articulated steering, skid-steering, etc. [4]. Among these, skid-steering is the unique steering approach for tracked drilling vehicles, realized by controlling the velocities of both tracks. The variation in the velocities per track pose the problem of slippage as well as terrain compression and shearing, which would be key factors in controlling the steering of vehicles [5]. Therefore, slippage must be included in the dynamics model, to improve the accuracy of locomotion in the trajectory control [6]. Moreover, external disturbances, e.g., Coulomb friction, must be included in the dynamics model, to represent the resistance force. Skidsteering may also cause loss of contact points between tracks and terrain, which further results in a deviation of the actual from the desired trajectory. This feature also poses difficulties in the motion control of tracked vehicles. Compared to road vehicles, the motion control of tracked drilling rigs calls for higher accuracy, due to the requirement for precise positioning of the hole centers, the constraint on the geo-fence boundary of the bench, and the need to avoid the drilled holes as obstacles [7].

Tracked vehicles are typical mechanical systems with nonholonomic constraints, for which motion control has become an intensive research field. Nonholonomic systems are characterized by nonintegrable constraint relations, i.e., constraints on the system generalized velocities [8]. One difficulty in the control of such systems is that the existence of a stabilizing smooth, time-invariant state feedback may not be guaranteed [9, 10], and hence, the methods of linear control theory are not applicable [11]. The demand for nonstandard feedback strategies for these nonlinear control problems thus arises. Compared to holonomic systems, controller design for their nonholonomic counterparts is more challenging, thus requiring special attention.

The trajectory-tracking control of tracked vehicles with nonholonomic constraints resembles that of wheeled vehicles, which have become a focus of re- 
search interest. In the literature, the trajectory-tracking controller design for mobile robots mainly falls into one of two categories: simple controller based only on the kinematic model [12, 13, 14, 6, 15]; and integration of the former with vehicle dynamics. As pointed out by Fierro and Lewis [16], the kinematic controller can simplify the nonholonomic tracking problem. However, the actual twist 1 is generated based on the assumption of "perfect twist tracking", which does not hold in reality. Formulation of the nonholonomic controller at the dynamic level makes it realistic and practical for industrial applications.

Numerous studies have been conducted on the controller design based on the integration of vehicle kinematics and dynamics. Sliding-mode control is a typical example, for nonholonomic constrained wheeled vehicles with two control inputs to asymptotically stabilize deviations from the desired trajectory [17, 18]. Adaptive fuzzy control [19] and robust adaptive control [20, 21, 22] were also reported for trajectory-tracking problems in the presence of system uncertainties and external disturbances. Hoang et al. introduced a neural network in their controller design to cope with uncertainties of the dynamics model of a nonholonomic mobile robot [23]. Klančar et al. derived a model-predictive trajectory-tracking control method for tracked mobile robots, enabling fast real-time implementation [24]. Fierro and Lewis proposed a back-stepping dynamics model into the kinematics of a tracked mobile robot [25]. The kinematic controller was designed to make the desired vehicle trajectory and the actual trajectory to converge. By using back-stepping, a torque controller was designed afterwards so that the difference between the desired vehicle velocities and actual velocities will converge to zero.

In general, compared with other nonlinear control methods, the structure of the back-stepping controller is less complicated to design. On the other hand, the complexity of other nonlinear control techniques poses difficulties to their implementation. For instance, the design of the adaptive controller involves the

\footnotetext{
${ }^{1}$ Velocity of a landmark point, preferably the center of mass, and angular velocity of a rigid body.
} 
determination of a couple of adaptive adjusting terms, thus resulting in design complexity.

Among the diverse types of control methodologies, PID control has gained acceptance, due to its advantages of lucid meaning, simplicity, and effectiveness. These features are significant in applications in industry, which makes PID control preferable to "complex control methods" [26]. PID control is capable of stabilizing nonlinear systems, as long as the system at hand is endowed with the skew-symmetry property (SSP) 27], whose proof for serial robots is available in the literature [28]:

Proposition 1 (The Skew-Symmetry Property). For the mechanical system model $\mathbf{M} \ddot{\mathbf{q}}+\mathbf{C}(\mathbf{q}, \dot{\mathbf{q}}) \dot{\mathbf{q}}=\mathbf{f}$, where $\mathbf{M}(\mathbf{q})$ is the inertia matrix, while $\mathbf{C}(\mathbf{q}, \dot{\mathbf{q}})$ is the matrix coefficient of Coriolis and centrifugal force 2 , with $\mathbf{q}$ denoting the vector of system generalized coordinates, the matrix difference $\mathbf{D}=\dot{\mathbf{M}}-2 \mathbf{C}$ is skew symmetric.

A detailed formulation of the dynamic equations for general multibody systems, and the proof of the skew-symmetry property were reported by From et al. [29].

In this paper, the computed-torque controller design, based on PID control, is proposed for tracked vehicles undergoing skid-steering on horizontal, hard terrain with nonholonomic constraints. The remainder of this paper is organized as follows: in Section 2, both the kinematics and dynamics models of the tracked vehicle are formulated. The nonholonomic constraint is derived using the theory of planar kinematics. In Section 3, the sensing system for the vehicle localization and navigation is described, based on a planar array of bi-axial accelerometers for pose and twist estimation of rigid bodies undergoing planar motion. Based upon the sensing system, the trajectory-tracking controller is designed using a modified PID computed-torque control scheme. The asymptotic stability of the system is proven by means of Lyapunov theory. The validation of the robustness

\footnotetext{
${ }^{2}$ The product $\mathbf{C}(\mathbf{q}, \dot{\mathbf{q}}) \dot{\mathbf{q}}$ yields the vector of generalized forces quadratic in the generalized velocity $\dot{\mathbf{q}}$, hence the name.
} 
and accuracy of the proposed control algorithm are reported in Section 4, via a simulation example. Finally, conclusions are drawn in Section 5.

\section{Modeling of Tracked Vehicles}

\subsection{Kinematics}

To model a tracked vehicle maneuvering on the ground, two coordinate frames are introduced, the vehicle-fixed frame $\mathcal{B}$, and the inertial frame $\mathcal{I}$, both illustrated in Fig. 11 Subscript $\mathcal{B}$ is used to indicate frame- $\mathcal{B}$ coordinates; when no subscript is included, the array representation is assumed in the inertial frame.

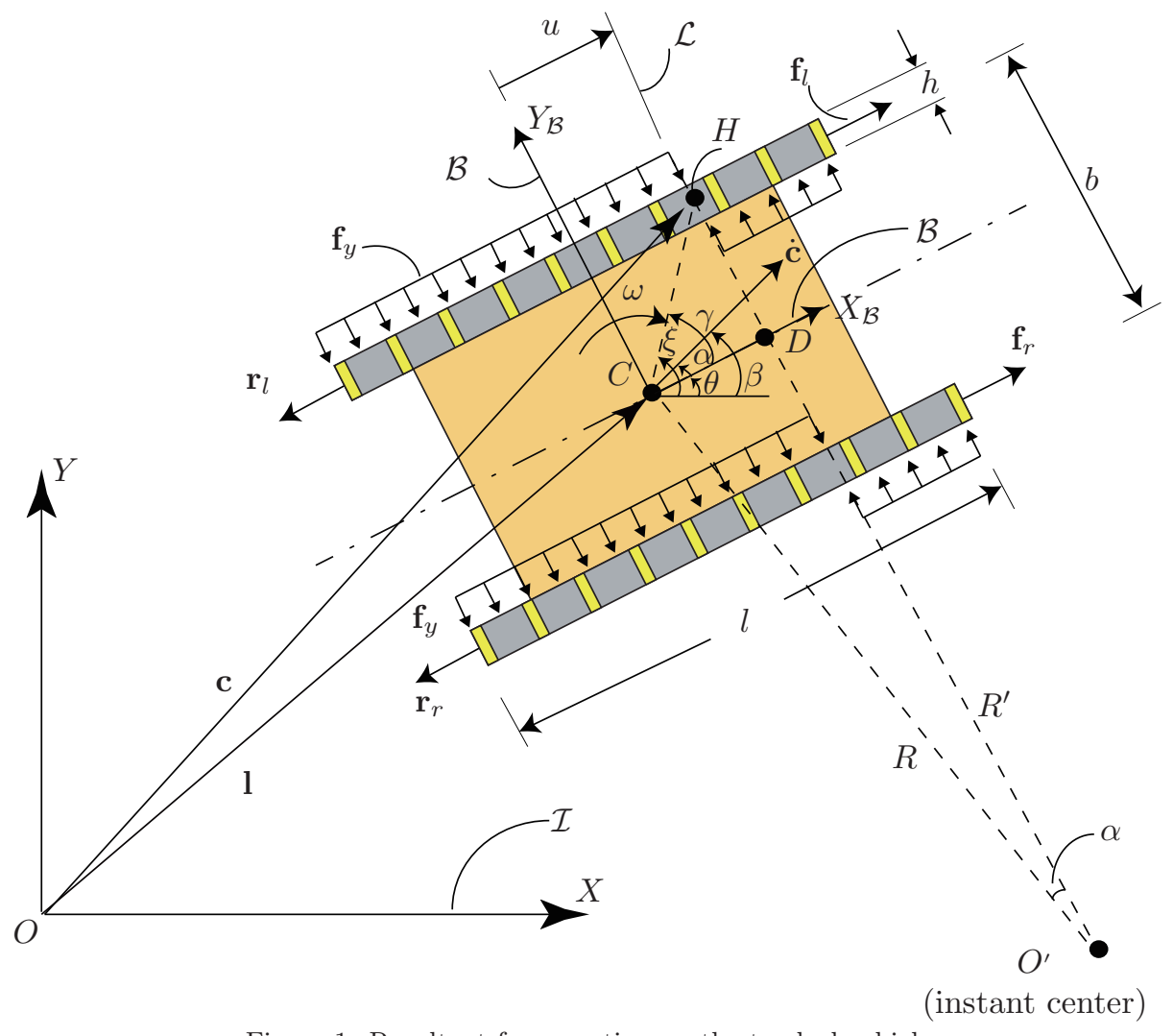

Figure 1: Resultant forces acting on the tracked vehicle

The velocity of point $C$ - the center of mass of the tracked vehicle, assumed 
to coincide with the centroid of the rectangle between the track 3 , the vehicle platform - is expressed in frame $\mathcal{B}$ as

$$
\begin{aligned}
{[\dot{\mathbf{c}}]_{\mathcal{B}} } & =\left[\begin{array}{c}
\dot{x}_{\mathcal{B}} \\
\dot{y}_{\mathcal{B}}
\end{array}\right]=\left[\begin{array}{l}
\|\dot{\mathbf{c}}\| \cos \alpha \\
\|\dot{\mathbf{c}}\| \sin \alpha
\end{array}\right] \\
\|\dot{\mathbf{c}}\| & =\frac{r}{2 \cos \alpha}\left[\omega_{l}\left(1-\sigma_{l}\right)+\omega_{r}\left(1-\sigma_{r}\right)\right]
\end{aligned}
$$

where the slip angle $\alpha$ is caused by the skid-steering turning maneuver of the vehicle, during which the instant center of rotation $O^{\prime}$ shifts a distance $u_{0}$ from the $Y_{\mathcal{B}}$-axis, $\omega_{l}$ and $\omega_{r}$ denoting the angular velocities of the left- and right trackdrive sprockets, respectively, $r$ the radius of the pitch circle of the track-drive sprockets.

The track slips for the left and right tracks are given by [30, 31]

$$
\begin{gathered}
\sigma_{l}=1-\frac{v_{l}}{r \omega_{l}}=1-\frac{\dot{x}_{\mathcal{B}}+(b / 2) \dot{\theta}}{r \omega_{l}} \\
\sigma_{r}=1-\frac{v_{r}}{r \omega_{r}}=1-\frac{\dot{x}_{\mathcal{B}}-(b / 2) \dot{\theta}}{r \omega_{r}}
\end{gathered}
$$

where $v_{l}$ and $v_{r}$ denote the actual forward speed of the left and right track, respectively.

The turning radius $R$ can be then obtained based on $\sigma_{l}$ and $\sigma_{r}$, i.e.,

$$
R=\frac{\|\dot{\mathbf{c}}\|}{|\omega|}=\frac{b}{2 \cos \alpha} \frac{\omega_{l}\left(1-\sigma_{l}\right)+\omega_{r}\left(1-\sigma_{r}\right)}{\omega_{l}\left(1-\sigma_{l}\right)-\omega_{r}\left(1-\sigma_{r}\right)}
$$

The $2 \times 2$ rotation matrix $\mathbf{Q}$ that carries $\mathcal{I}$ into $\mathcal{B}$ takes the form

$$
\mathbf{Q}=\left[\begin{array}{cc}
c \theta & -s \theta \\
s \theta & c \theta
\end{array}\right]
$$

where $c(\cdot)$ and $s(\cdot)$ stand for $\cos (\cdot)$ and $\sin (\cdot)$, respectively.

\footnotetext{
${ }^{3}$ Most likely this will not be the case in practice. The assumption is adopted here to simplify the formulation.
} 
Hence, the velocity of point $C$ in $\mathcal{I}$ is obtained, from $[\dot{\mathbf{c}}]_{\mathcal{B}}$, by means of the rotation matrix $\mathbf{Q}$ :

$$
\begin{aligned}
\dot{\mathbf{c}} & =\mathbf{Q}[\dot{\mathbf{c}}]_{\mathcal{B}}=\left[\begin{array}{l}
\dot{x} \\
\dot{y}
\end{array}\right]=\|\dot{\mathbf{c}}\|\left[\begin{array}{l}
c \theta c \alpha-s \theta s \alpha \\
s \theta c \alpha+c \theta s \alpha
\end{array}\right] \\
& =\frac{r}{2}\left[\omega_{l}\left(1-\sigma_{l}\right)+\omega_{r}\left(1-\sigma_{r}\right)\right]\left[\begin{array}{l}
c \theta-s \theta \tan \alpha \\
s \theta+c \theta \tan \alpha
\end{array}\right]
\end{aligned}
$$

Now, incorporating the expression for the angular velocity in the above relation, the complete kinematic model in $\mathcal{I}$ is given by

$$
\begin{aligned}
& \dot{x}=\frac{r}{2}\left[\omega_{l}\left(1-\sigma_{l}\right)+\omega_{r}\left(1-\sigma_{r}\right)\right][c \theta-s \theta \tan \alpha] \\
& \dot{y}=\frac{r}{2}\left[\omega_{l}\left(1-\sigma_{l}\right)+\omega_{r}\left(1-\sigma_{r}\right)\right][s \theta+c \theta \tan \alpha] \\
& \dot{\theta}=\frac{r}{b}\left[\omega_{l}\left(1-\sigma_{l}\right)-\omega_{r}\left(1-\sigma_{r}\right)\right]
\end{aligned}
$$

\subsection{The nonholonomic constraint}

As shown in Fig. 1 the instant center $O^{\prime}$ lies on line $\mathcal{L}$, offset from point $C$ by the amount $u(t)$, in the $X_{\mathcal{B}}$-direction. Let $H$, the intersection between $\mathcal{L}$ and the centerline of the left track, be the contact point of the left track with the terrain, of position vector $\mathbf{l}$ in frame $\mathcal{I}$. $\boldsymbol{\Omega}$ denotes the $2 \times 2$ angular-velocity matrix, that maps the relative position vector $\mathbf{l}-\mathbf{c}$ into the relative velocity $\dot{\mathrm{i}}-\dot{\mathbf{c}}$, namely

$$
\mathrm{i}-\dot{\mathbf{c}}=\Omega(\mathbf{l}-\mathbf{c})
$$

where $\boldsymbol{\Omega}$ is given by 32$]$

$$
\boldsymbol{\Omega}=\dot{\mathbf{Q}} \mathbf{Q}^{T}=\dot{\theta} \mathbf{E}
$$

with $\mathbf{E}$ representing a rotation in the plane of motion by an angle of $90^{\circ}$ counterclockwise, namely,

$$
\mathbf{E}=\left[\begin{array}{cc}
0 & -1 \\
1 & 0
\end{array}\right]
$$

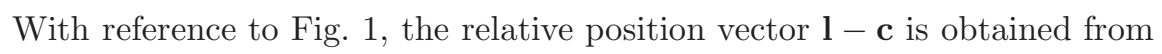


the geometry of the triangle $C D H$, namely

$$
\mathbf{l}-\mathbf{c}=\sqrt{(b / 2)^{2}+u^{2}}\left[\begin{array}{l}
\cos \xi \\
\sin \xi
\end{array}\right]
$$

Substitution of eqs. (8) and (9) into eq. (7) yields

$$
\mathbf{i}=\dot{\mathbf{c}}+\dot{\theta} \sqrt{(b / 2)^{2}+u^{2}}\left[\begin{array}{c}
-\sin \xi \\
\cos \xi
\end{array}\right]
$$

Now, if $[\mathbf{l}]_{\mathcal{B}}=\left[x_{\mathcal{B}}^{H}, y_{\mathcal{B}}^{H}\right]^{T}$, then the velocity of $H$ in $\mathcal{B}$ is obtained as

$$
[\mathbf{i}]_{\mathcal{B}}=\left[\begin{array}{c}
\dot{x}_{\mathcal{B}}^{H} \\
\dot{y}_{\mathcal{B}}^{H}
\end{array}\right]=\mathbf{Q}^{T} \mathbf{i}=\left[\begin{array}{c}
\dot{x} \cos \theta+\dot{y} \sin \theta \\
-\dot{x} \sin \theta+\dot{y} \cos \theta
\end{array}\right]+\dot{\theta} \sqrt{(b / 2)^{2}+u^{2}}\left[\begin{array}{c}
-\sin \gamma \\
\cos \gamma
\end{array}\right]
$$

From the distribution of the lateral resistance force $F_{y}$ - the friction force on the tracks along the lateral direction-we can observe that the track velocities along $\mathcal{L}$ vanish in the lateral direction, where the sign of $F_{y}$ changes [33]. Hence, the second component of $[\mathbf{i}]_{\mathcal{B}}$ in eq. (12) vanishes. Indeed,

$$
\begin{aligned}
\dot{y}_{\mathcal{B}}^{H} & =-\dot{x} \sin \theta+\dot{y} \cos \theta+\dot{\theta} \sqrt{(b / 2)^{2}+u^{2}} \cos \gamma \\
& =-\dot{x} \sin \theta+\dot{y} \cos \theta+\dot{\theta} u=0
\end{aligned}
$$

The above equation is a kinematic constraint, whose nonholonomy can be readily proven $[34]$.

Substituting eqs. (3) and (6a) - (6c) into (13) yields

$$
u=-\operatorname{sgn}(\dot{\theta}) R \sin \alpha
$$

with $\operatorname{sgn}(\cdot)$ representing the signum function:

$$
\operatorname{sgn}(x)= \begin{cases}+1, & \text { if } x>0 \\ 0, & \text { if } x=0 \\ -1, & \text { if } x<0\end{cases}
$$


In order to obtain a dimensionally homogenous mass matrix 4 for the dynamics model, the concept of radius of gyration, defined as $L=\sqrt{I / m}$, is recalled from basic mechanics and used as a characteristic length, with $m$ and $I$ denoting the mass of the vehicle and its moment of inertia about its c.o.m., respectively. More details will be given in Subsec. 2.3. Now a set of generalized coordinates $\mathbf{q}$ is defined as

$$
\mathbf{q}=\left[\begin{array}{c}
x \\
y \\
L \theta
\end{array}\right]
$$

which is dimensionally homogeneous, with all entries carrying units of length.

The nonholonomic constraint in eq. (13) can be rewritten in array form, namely,

$$
\mathbf{a}^{T}(\mathbf{q}) \dot{\mathbf{q}}=0, \quad \mathbf{a}(\mathbf{q})=\left[\begin{array}{c}
-s \theta \\
c \theta \\
u / L
\end{array}\right]
$$

The form of the nonholonomic constraint in eq. (16), linear in $\dot{\mathbf{q}}$, is called Pfaffian [35].

Let $\mathbf{S}(\mathbf{q}) \in \mathbb{R}^{3 \times 2}$ be a full-rank matrix, whose columns are orthogonal to $\mathbf{a}(\mathbf{q})$, i.e. 5 ,

$$
\mathbf{S}^{T}(\mathbf{q}) \mathbf{a}(\mathbf{q})=\mathbf{0}
$$

One possible form of $\mathbf{S}(\mathbf{q})$ is given below

$$
\mathbf{S}=\left[\begin{array}{cc}
c \theta & u s \theta / L \\
s \theta & -u c \theta / L \\
0 & 1
\end{array}\right]
$$

\footnotetext{
${ }^{4} \mathrm{~A}$ homogeneous matrix is needed in order to obtain a physically meaningful difference $\dot{\mathbf{M}}-2 \mathbf{C}$ in Subsection 2.3

${ }^{5}$ Vector $\mathbf{a}(\mathbf{q})$ can be regarded as proportional to the cross product of the two columns of $\mathbf{S}(\mathbf{q})$.
} 
According to Fierro and Lewis [25], an auxiliary vector $\boldsymbol{v}(t) \in \mathbb{R}^{2}$ can be defined based on eqs. (16) and (17), namely

$$
\dot{\mathbf{q}}=\mathbf{S}(\mathbf{q}) \boldsymbol{v}(t)
$$

where $\boldsymbol{v}(t)=\left[\begin{array}{ll}v & \dot{\theta}\end{array}\right]^{T}$, with $v$ and $\dot{\theta}$ representing the speed and the angular velocity of the vehicle, respectively.

\subsection{Mathematical Model}

As illustrated in Fig. (1) $\mathbf{f}_{l}$ and $\mathbf{f}_{r}$ denote the tractive forces applied on the two tracks by the ground, with magnitudes $F_{l}$ and $F_{r}$, respectively, $\mathbf{r}_{l}$ and $\mathbf{r}_{r}$ being the longitudinal resistance forces exerted by the terrain on the tracks, whose magnitudes are $R_{l}$ and $R_{r}$, respectively. Moreover, $\mathbf{f}_{y}$ is the lateral resistance force, with a magnitude $F_{y}$. The dynamics model of the tracked vehicle is formulated in $\mathcal{B}$ as

$$
\begin{aligned}
m \ddot{x}_{\mathcal{B}} & =F_{l}+F_{r}-R_{l}-R_{r} \\
m \ddot{y}_{\mathcal{B}} & =F_{y} \\
I \ddot{\theta} & =M-M_{r}
\end{aligned}
$$

where $M$ denotes magnitude of the turning moment, $M_{r}$ being the magnitude of its resistive counterpart, produced by the lateral resistance force $\mathbf{f}_{y}$.

We notice that eq. (20c) has units of moment of inertia, while eqs. (20a) and (20b have units of mass. With the purpose of obtaining a dimensionally homogeneous mass matrix, the characteristic length $L=\sqrt{I / m}$ is introduced. Upon dividing both sides of eq. (20c) by $L$, we obtain a new equation with units of force, namely,

$$
\frac{I}{L^{2}} L \ddot{\theta}=\frac{M}{L}-\frac{M_{r}}{L}
$$

The detailed expressions for the dynamics model are given below. 


\subsubsection{The Tractive Force}

The maximum tractive force developed by a track is determined by the area $A$ of the tracks in contact with the terrain, and the maximum shear strength of the terrain $\tau_{m}$ [36], i.e.,

$$
F_{m}=A \tau_{m}=A(c+p \tan \varphi)=A c+\frac{W}{2} \tan \varphi
$$

in which $A=h l$, while $c$ and $\varphi$ represent the apparent cohesion $\left(\mathrm{N} / \mathrm{m}^{2}\right)$ and the angle of internal shearing resistance of the terrain, respectively, $p$ being the normal pressure beneath the track, and $W$ the weight of the vehicle.

For a specified track slip $i$ at a given instant, the tractive force of a track is calculated as [4]

$$
F=F_{m}\left[1-\frac{K}{i l}\left(1-e^{-i l / K}\right)\right]
$$

in which $K$ represents the soil shear deformation modulus with units of $\mathrm{m}$.

\subsubsection{The Longitudinal and Lateral Resistance Forces}

Assuming an evenly distributed load on both tracks, the longitudinal motion resistance of the left and the right tracks, $R_{l}$ and $R_{r}$, are calculated as

$$
R_{l}=R_{r}=\mu_{l} \frac{W}{2}
$$

The resistance force in the lateral direction is given by

$$
F_{y}=2 \operatorname{sgn}(\omega) \mu_{t}\left[\int_{-l / 2}^{u} \frac{W}{2 l} d x-\int_{u}^{l / 2} \frac{W}{2 l} d x\right]=2 \operatorname{sgn}(\omega) \mu_{t} u \frac{W}{l}
$$

where $W=m g$ represents the vehicle weight, $\mu_{l}$ and $\mu_{t}$ being the coefficients of motion resistance of the vehicle in the longitudinal and lateral directions, respectively. 


\subsubsection{The Turning Moment and Moment of Turning Resistance}

The turning moment is produced by the tractive forces and the resistance forces in the longitudinal direction, namely,

$$
M=\left(F_{l}-R_{l}\right) \frac{b}{2}-\left(F_{r}-R_{r}\right) \frac{b}{2}=\left(F_{l}-F_{r}\right) \frac{b}{2}
$$

The moment of turning resistance is given by

$$
M_{r}=2 \operatorname{sgn}(\omega) \mu_{t}\left(\int_{-l / 2}^{u} \frac{W}{2 l} x d x-\int_{u}^{l / 2} \frac{W}{2 l} x d x\right)=\operatorname{sgn}(\omega) \mu_{t} \frac{W}{l}\left(\|\dot{\mathbf{c}}\|^{2} \frac{\sin ^{2} \alpha}{\omega^{2}}-\frac{l^{2}}{4}\right)
$$

By the same token, the mathematical model in $\mathcal{I}$ can be obtained by means of the transformation matrix $\mathbf{Q}$ of eq. (4), which leads to

$$
\begin{aligned}
m \ddot{x} & =\left(F_{l}+F_{r}\right) c \theta-2 R_{l} c \theta-F_{y} s \theta \\
m \ddot{y} & =\left(F_{l}+F_{r}\right) s \theta-2 R_{l} s \theta+F_{y} c \theta \\
\frac{I}{L^{2}} L \ddot{\theta} & =\left(F_{l}-F_{r}\right) \frac{b}{2 L}-\operatorname{sgn}(\omega) \mu_{t} \frac{W}{L l}\left[\|\dot{\mathbf{c}}\|^{2} \frac{\sin ^{2} \alpha}{\omega^{2}}-\frac{l^{2}}{4}\right]
\end{aligned}
$$

Rearranging the above equations into array form and taking into account the nonholonomic constraint in eq. (16) yields the mathematical model of interest, namely,

$$
\mathbf{M}(\mathbf{q}) \ddot{\mathbf{q}}+\mathbf{f}_{1}(\dot{\mathbf{q}})+\mathbf{f}_{2}(\mathbf{q}, \dot{\mathbf{q}})+\mathbf{a}(\mathbf{q}) \lambda=\mathbf{B}(\mathbf{q}) \tau
$$

where $\mathbf{a}(\mathbf{q})$ is defined in eq. (16) and

$$
\begin{aligned}
& \mathbf{M}(\mathbf{q})=\left[\begin{array}{ccc}
m & 0 & 0 \\
0 & m & 0 \\
0 & 0 & I / L^{2}
\end{array}\right] \equiv m \mathbf{1}_{3 \times 3}, \quad \mathbf{f}_{1}(\mathbf{q})=\left[\begin{array}{c}
2 R_{l} c \theta \\
2 R_{l} s \theta \\
0
\end{array}\right], \quad \mathbf{f}_{2}(\mathbf{q}, \dot{\mathbf{q}})=\left[\begin{array}{c}
F_{y} s \theta \\
-F_{y} c \theta \\
M_{r} / L
\end{array}\right] \\
& \mathbf{B}(\mathbf{q})=\left[\begin{array}{cc}
c \theta & c \theta \\
s \theta & s \theta \\
b / 2 L & -b / 2 L
\end{array}\right], \quad \ddot{\mathbf{q}}=\left[\begin{array}{c}
\ddot{x} \\
\ddot{y} \\
L \ddot{\theta}
\end{array}\right], \quad \boldsymbol{\tau}=\left[\begin{array}{c}
F_{l} \\
F_{r}
\end{array}\right]
\end{aligned}
$$


in which $\mathbf{1}_{3 \times 3}$ denotes the $3 \times 3$ identity matrix, $\lambda$ is the constraint force and $\mathbf{M}(\mathbf{q})=m \mathbf{1}_{3 \times 3}$ is the (constant) mass matrix.

Differentiating both sides of eq. (19) w.r.t. time leads to

$$
\ddot{\mathbf{q}}=\dot{\mathbf{S}}(\mathbf{q}) \boldsymbol{v}(t)+\mathbf{S}(\mathbf{q}) \dot{\boldsymbol{v}}(t)
$$

Further, substituting eqs. (19) and (30) into eq. (29) and left-multiplying both sides of the resulting equation by $\mathbf{S}^{T}$ yields,

$$
\widetilde{\mathbf{M}}(\mathbf{q}) \dot{\boldsymbol{v}}(t)+\widetilde{\mathbf{C}}(\mathbf{q}, \dot{\mathbf{q}}) \boldsymbol{v}(t)+\tilde{\mathbf{f}}_{1}(\dot{\mathbf{q}})+\tilde{\mathbf{f}}_{2}(\mathbf{q}, \dot{\mathbf{q}})=\widetilde{\mathbf{B}}(\mathbf{q}) \boldsymbol{\tau}
$$

where

$$
\begin{aligned}
\widetilde{\mathbf{M}}(\mathbf{q}) & =\mathbf{S}^{T} \mathbf{M} \mathbf{S}=\left[\begin{array}{cc}
m & 0 \\
0 & \left(m u^{2}+I\right) / L^{2}
\end{array}\right]=m\left[\begin{array}{cc}
1 & 0 \\
0 & u^{2} / L^{2}+1
\end{array}\right] \\
\widetilde{\mathbf{C}}(\mathbf{q}, \dot{\mathbf{q}}) & =\mathbf{S}^{T} \mathbf{M} \dot{\mathbf{S}}=\left[\begin{array}{cc}
0 & m u \dot{\theta} / L \\
-m u \dot{\theta} / L & m u \dot{u} / L^{2}
\end{array}\right] \\
\widetilde{\mathbf{B}} & =\mathbf{S}^{T} \mathbf{B}=\left[\begin{array}{cc}
1 & 1 \\
b / 2 L & -b / 2 L
\end{array}\right], \quad \tilde{\mathbf{f}}_{1}=\mathbf{S}^{T} \mathbf{f}_{1}=\left[\begin{array}{c}
2 R_{l} \\
0
\end{array}\right] \\
\tilde{\mathbf{f}}_{2} & =\mathbf{S}^{T} \mathbf{f}_{2}=\left[\begin{array}{cc}
0 \\
\left(F_{y} u+M_{r}\right) / L
\end{array}\right]=\left[\begin{array}{c}
0 \\
\operatorname{sgn}(\omega) \mu_{t}(W / L l)\left(3 u^{2}-l^{2} / 4\right)
\end{array}\right]
\end{aligned}
$$

Notice that the second term of the RHS of eq. (31) is quadratic in the generalized velocities, reason why this term is known as the Coriolis-force term, its matrix coefficient $\widetilde{\mathbf{C}}(\mathbf{q}, \dot{\mathbf{q}})$ being known as the "matrix of Coriolis forces".

The skew-symmetry property for the case at hand is proven below:

$$
\begin{aligned}
\dot{\widetilde{\mathbf{M}}}-2 \widetilde{\mathbf{C}} & =\dot{\mathbf{S}}^{T} \mathbf{M S}+\mathbf{S}^{T} \dot{\mathbf{M}} \mathbf{S}+\mathbf{S}^{T} \mathbf{M} \dot{\mathbf{S}}-2 \mathbf{S}^{T} \mathbf{M} \dot{\mathbf{S}} \\
& =\dot{\mathbf{S}}^{T} \mathbf{M S}-\left(\dot{\mathbf{S}}^{T} \mathbf{M S}\right)^{T}+\mathbf{S}^{T} \underbrace{\dot{\mathbf{M}}}_{\mathbf{O}} \mathbf{S} \\
& =\dot{\mathbf{S}}^{T} \mathbf{M S}-\left(\dot{\mathbf{S}}^{T} \mathbf{M S}\right)^{T}
\end{aligned}
$$


thereby proving that $\dot{\widetilde{\mathbf{M}}}-2 \widetilde{\mathbf{C}}$ is skew-symmetric.

\section{Controller Design}

The complete mathematical model, given by eqs.(19) and(31), is reproduced below for quick reference:

$$
\begin{aligned}
& \dot{\mathbf{q}}=\mathbf{S}(\mathbf{q}) \boldsymbol{v}(t) \\
& \widetilde{\mathbf{M}}(\mathbf{q}) \dot{\boldsymbol{v}}(t)+\widetilde{\mathbf{C}}(\mathbf{q}, \dot{\mathbf{q}}) \boldsymbol{v}(t)+\tilde{\mathbf{f}}_{1}(\dot{\mathbf{q}})+\tilde{\mathbf{f}}_{2}(\mathbf{q}, \dot{\mathbf{q}})=\widetilde{\mathbf{B}}(\mathbf{q}) \boldsymbol{\tau}
\end{aligned}
$$

Defining the control input $\mathbf{u}_{c}=\widetilde{\mathbf{B}}(\mathbf{q}) \boldsymbol{\tau}$ and $\mathbf{x}_{t}=\left[\begin{array}{ll}\mathbf{q}^{T} & \boldsymbol{v}^{T}\end{array}\right]^{T}$, the mathematical model above can be rewritten in state-variable form as

$$
\dot{\mathbf{x}}_{t}=\mathbf{f}\left(\mathrm{x}_{t}\right)+\boldsymbol{\Gamma}\left(\mathrm{x}_{t}\right) \mathbf{u}_{c}
$$

where

$$
\mathbf{f}\left(\mathbf{x}_{t}\right)=\left[\begin{array}{c}
\mathbf{S} \boldsymbol{v} \\
-\widetilde{\mathbf{M}}^{-1}\left(\widetilde{\mathbf{C}} \boldsymbol{v}+\tilde{\mathbf{f}}_{1}+\tilde{\mathbf{f}}_{2}\right)
\end{array}\right], \quad \boldsymbol{\Gamma}\left(\mathbf{x}_{t}\right)=\left[\begin{array}{c}
\mathbf{O}_{3 \times 2} \\
\widetilde{\mathbf{M}}^{-1}
\end{array}\right]
$$

with $\mathbf{O}_{3 \times 2}$ denoting the $3 \times 2$ zero matrix.

The design of the trajectory-tracking controller for the governing equations of the above system is described below. Additionally, the design of the onboard sensing system is introduced, whose measurements will be fed back into the controller, to realize the closed-loop control.

\subsection{The Sensing System for Vehicle Localization and Navigation}

Localization and navigation of the autonomous mobile vehicle in motion is usually realized by an on-board Global Positioning System (GPS) capable of providing an accurate estimate of the real-time position of the vehicle [2]. As described by Hennessy et al. [7], the position of a blasthole drill is measured by a GPS, which uses a receiver antenna at the top of the drill rig mast. A highprecision GPS (HPGPS), such as Topcon D-GPS, is a good candidate. Two 


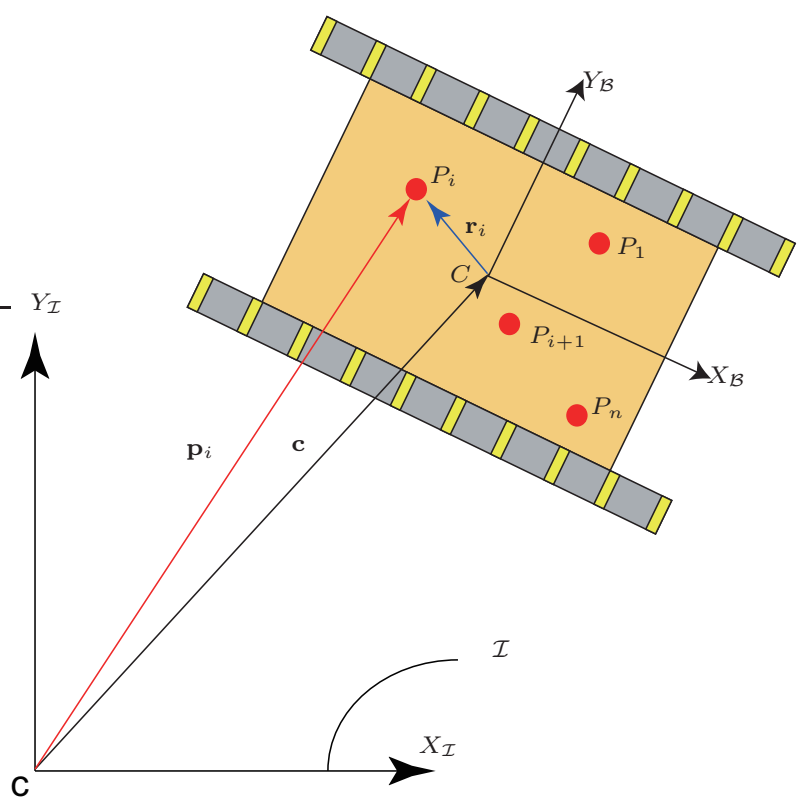

Figure 2: Tracked vehicle carrying an array of planar accelerometers

track encoders measure the angular velocities of the drive sprockets. Tracked vehicles instrumented with a HPGPS offer great benefits in providing accurate information of the actual path of motion; however, HPGPS do not ensure that the tracked vehicles will follow the prescribed path without motion control [37]. Moreover, GPSs are not fail-safe. Hence, Inertial Measurement Units (IMU) and dead-reckoning sensors, such as wheel encoders, integrated for data-fusion, appear as a better option. The use of redundant sensors not only increases the navigation reliability and precision, but also enables integrity requirements [1].

The sensing system mounted on the blasthole drill under study is responsible for the vehicle localization and navigation. A tracked vehicle moving on a horizontal plane carrying $n$ accelerometers $\left\{P_{i}\right\}_{1}^{n}$ is illustrated in Fig. 2, The reference point $C$ is the center of mass of the tracked vehicle. Vector $\mathbf{p}_{i}$ denotes the position of the $i$ th accelerometer, located at point $P_{i}$. From Fig. 2, vector $\mathbf{r}_{i}$ is defined as $\mathbf{r}_{i}=\mathbf{p}_{i}-\mathbf{c}$, for $i=1,2,3$, as three bi-axial accelerometers 
are employed6. Bi-axial accelerometers, by their nature, offer high out-of-plane stiffness, while providing sensitivity to acceleration of the proof-mass in the working plane. The rapid development of MEMS (microelectromechanical systems) techniques eases the fabrication of bi-axial accelerometers and promotes their applications in industry [38]. The three bi-axial accelerometers form an IMU, which enables the estimation of the pose and the twist of the blasthole drill in the moving plane, since the out-of-plane motion is not significant.

Instead of the regular IMU composed of both accelerometers and gyroscopes, a gyroscope-free IMU (GF-IMU), a.k.a. accelerometer strapdown, will be employed. One typical configuration of the sensing system is illustrated in Fig. 3 , The IMU and the pertinent estimation algorithm are described in full detail in a previous publication [39].

\subsection{Modified PID Computed-Torque Control Integrated with the Backstepping} Method

A modified PID computed-torque controller will be designed for this nonlinear system, as explained below.

In this trajectory-tracking control problem, two vehicle poses are defined: the reference (target) pose $\mathbf{q}_{r}$, and the actual pose under slip, $\mathbf{q}_{s}$. The reference trajectory is described by the equation below:

$$
\dot{\mathbf{q}}_{r}=\left[\begin{array}{c}
\dot{x}_{r} \\
\dot{y}_{r} \\
\dot{\theta}_{r}
\end{array}\right]=\left[\begin{array}{cc}
\cos \theta_{r} & 0 \\
\sin \theta_{r} & 0 \\
0 & L
\end{array}\right]\left[\begin{array}{c}
v_{r} \\
\omega_{r}
\end{array}\right]
$$

As explained in Sec. 1, due to the skid-steering turning maneuver of the tracked vehicle, slippage is inevitable in practice. Hence, when taking into account the influence of slippage, the actual orientation of the tracked vehicle

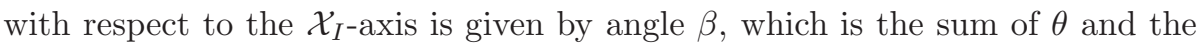

\footnotetext{
${ }^{6}$ Two biaxial accelerometers suffice, a third one is included for redundancy, and hence, robustness to measurement errors.
} 


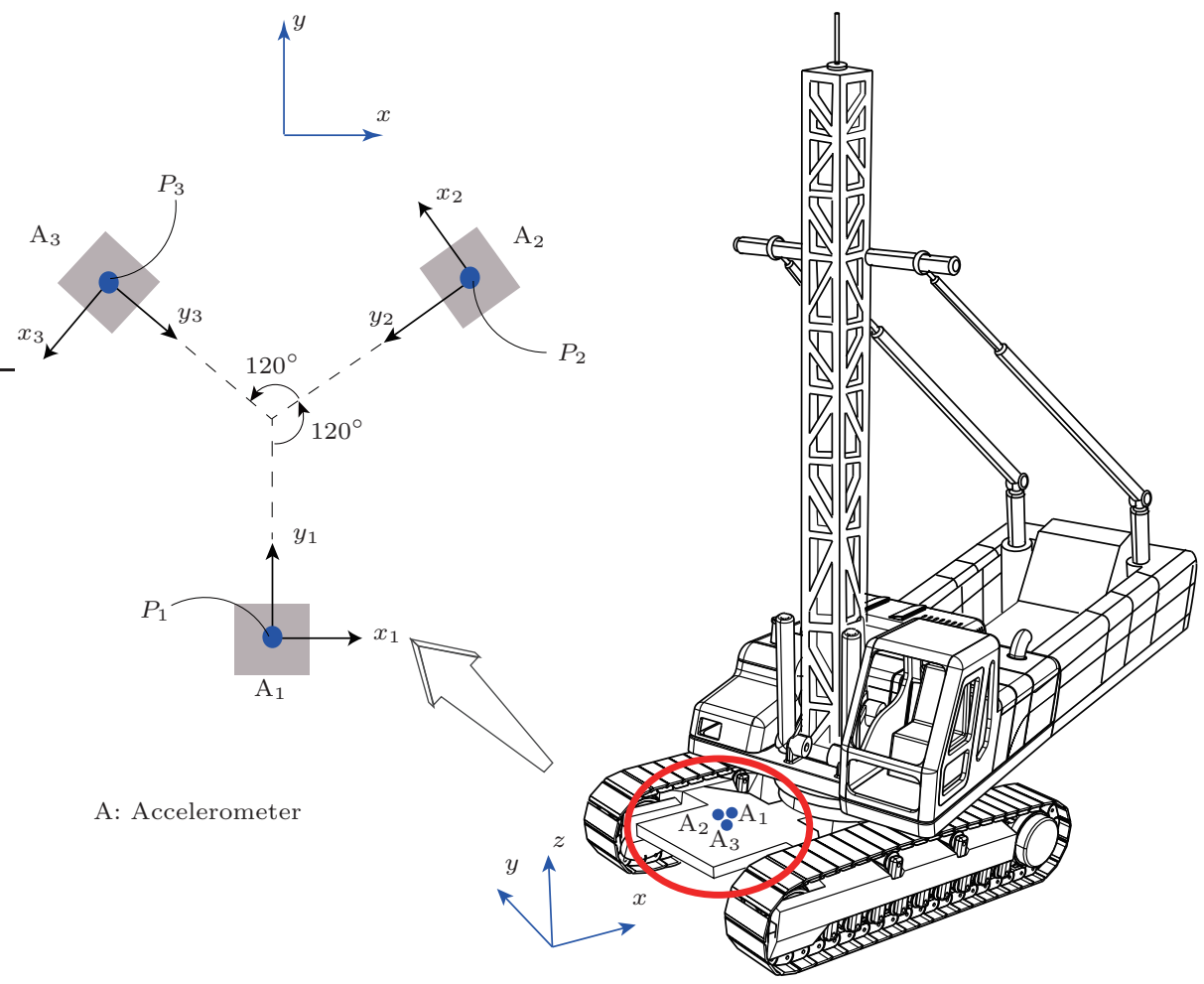

Figure 3: Biaxial accelerometer strapdown for the estimation of pose and twist of blastholedrill platform

slip angle $\alpha$, as illustrated in Fig. 1 Hence, the actual pose of the vehicle with slip $\mathbf{q}_{s}$ is expressed as $\left[\begin{array}{lll}x & y & L\end{array}\right]^{T}$, instead of $\left[\begin{array}{lll}x & y & L\end{array}\right]^{T} \mathrm{~T}$. The difference between these two poses, defined as the pose tracking error $\mathbf{e}_{p}$, is expressed in the local frame $\mathcal{B}[40]$ as

$$
\left[\mathbf{e}_{p}\right]_{\mathcal{B}}=\left[\begin{array}{c}
e_{1} \\
e_{2} \\
e_{3}
\end{array}\right]_{\mathcal{B}}=\left[\mathbf{R}\left(\mathbf{q}_{r}-\mathbf{q}_{s}\right)\right]_{\mathcal{B}}=\left[\begin{array}{ccc}
c \theta & s \theta & 0 \\
-s \theta & c \theta & 0 \\
0 & 0 & 1
\end{array}\right]\left[\begin{array}{c}
x_{r}-x \\
y_{r}-y \\
L\left(\theta_{r}-\beta\right)
\end{array}\right]
$$

\footnotetext{
${ }^{7}$ Due to the existence of the slip angle $\alpha$, the orientation of the actual velocity of the vehicle $\dot{\mathbf{c}}$ is not along the longitudinal direction of the vehicle body any more. The difference between $\dot{\mathbf{c}}$ and the vehicle longitudinal direction is the slip angle $\alpha$. Therefore, in the inertial frame $\mathcal{I}$, the real-time orientation of the vehicle is $\beta$, which is the sum of angles $\theta$ and $\alpha$.
} 
where $\mathbf{R}$ is a $3 \times 3$ rotation matrix carrying the three-dimensional pose error from $\mathcal{B}$ into $\mathcal{I}$. The three components of $\left[\mathbf{e}_{p}\right]_{\mathcal{B}}$ represent the forward, lateral and angular errors, respectively [41, 42].

The derivative of the pose tracking error is thus readily obtained:

$$
\left[\dot{\mathbf{e}}_{p}\right]_{\mathcal{B}}=\left[\begin{array}{c}
\dot{e}_{1} \\
\dot{e}_{2} \\
\dot{e}_{3}
\end{array}\right]=\left[\begin{array}{c}
-\cos \alpha \\
-\cos \alpha \tan \alpha \\
0
\end{array}\right] v+\left[\begin{array}{c}
e_{2} \\
-e_{1} \\
-1
\end{array}\right] \omega+\left[\begin{array}{c}
v_{r} \cos \left(e_{3} / L+\alpha\right) \\
v_{r} \sin \left(e_{3} / L+\alpha\right) \\
L\left(\omega_{r}-\dot{\alpha}\right)
\end{array}\right]
$$

The auxiliary twist control input $\mathbf{v}_{c}$ is given by [40]

$$
\mathbf{v}_{c}=\left[\begin{array}{c}
v_{r} \cos \left(e_{3} / L\right)+k_{x} e_{1} \\
\omega_{r}+v_{r}\left[k_{y} e_{2}+k_{\theta} \sin \left(e_{3} / L\right)\right]
\end{array}\right]
$$

in which $k_{x}, k_{y}$ and $k_{\theta}$ are positive constants.

Further, the time derivative of $\mathbf{v}_{c}$ is obtained as

$$
\dot{\mathbf{v}}_{c}=\left[\begin{array}{c}
\dot{v}_{r} \cos \left(e_{3} / L\right) \\
\dot{\omega}_{r}+\dot{v}_{r}\left[k_{y} e_{2}+k_{\theta} \sin \left(e_{3} / L\right)\right]
\end{array}\right]+\left[\begin{array}{ccc}
k_{x} & 0 & -v_{r} \sin \left(e_{3} / L\right) / L \\
0 & k_{y} v_{r} & k_{\theta} v_{r} \cos \left(e_{3} / L\right) / L
\end{array}\right]\left[\begin{array}{c}
\dot{e}_{1} \\
\dot{e}_{2} \\
\dot{e}_{3}
\end{array}\right]
$$

Assuming that the reference twist $\mathbf{v}_{r}$ is constant, eq. (39) can be rewritten as

$$
\dot{\mathbf{v}}_{c}=\left[\begin{array}{ccc}
k_{x} & 0 & -v_{r} \sin \left(e_{3} / L\right) / L \\
0 & k_{y} v_{r} & k_{\theta} v_{r} \cos \left(e_{3} / L\right) / L
\end{array}\right]\left[\begin{array}{c}
\dot{e}_{1} \\
\dot{e}_{2} \\
\dot{e}_{3}
\end{array}\right]
$$

Now we define the twist-tracking error $\mathbf{e}_{v}$ as the difference between the desired twist control input and the actual twist, i.e.,

$$
\mathbf{e}_{v}=\mathbf{v}_{c}-\mathbf{v}
$$

Furthermore, based on the above definition, we propose a modified PID 
computed-torque controller, formulated as

$$
\widetilde{\mathbf{B}} \tau=\widetilde{\mathbf{M}}\left(\dot{\mathbf{v}}_{c}+k_{p} \mathbf{e}_{v}\right)+\widetilde{\mathbf{C}} \mathbf{v}_{c}+\tilde{\mathbf{f}}_{1}+\tilde{\mathbf{f}}_{2}+k_{p} \mathbf{e}_{v}+k_{i} \int \mathbf{e}_{v} d t+k_{d} \dot{\mathbf{e}}_{v}
$$

where $k_{p}, k_{i}$ and $k_{d}$ are the proportional, integral and derivative gains, respectively, all positive.

Substituting eq. (42) into eq. (33b) leads to

$$
\left(\widetilde{\mathbf{M}}+k_{d} \mathbf{1}\right) \dot{\mathbf{e}}_{v}+\left(\widetilde{\mathbf{C}}+\widetilde{\mathbf{M}} k_{p} \mathbf{1}+k_{p} \mathbf{1}\right) \mathbf{e}_{v}+k_{i} \int \mathbf{e}_{v} d t=0
$$

To study the stability of the system (43), a positive-definite Lyapunov function candidate is chosen:

$$
V=\frac{1}{2} \mathbf{e}_{v}^{T}\left(\widetilde{\mathbf{M}}+k_{d} \mathbf{1}\right) \mathbf{e}_{v}+\frac{1}{2}\left(\int \mathbf{e}_{v} d t\right)^{T} k_{i}\left(\int \mathbf{e}_{v} d t\right)
$$

Differentiating eq. (44) with respect to time, and considering the closed-loop dynamics model (43), yields

$$
\begin{aligned}
\dot{V} & =\mathbf{e}_{v}^{T}\left[\left(\widetilde{\mathbf{M}}+k_{d} \mathbf{1}\right) \dot{\mathbf{e}}_{v}+\frac{1}{2} \dot{\widetilde{\mathbf{M}}} \mathbf{e}_{v}+k_{i}\left(\int \mathbf{e}_{v} d t\right)\right] \\
& =\mathbf{e}_{v}^{T}\left[-\left(\widetilde{\mathbf{C}}+\widetilde{\mathbf{M}} k_{p} \mathbf{1}+k_{p} \mathbf{1}\right) \mathbf{e}_{v}-k_{i}\left(\int \mathbf{e}_{v} d t\right)+\frac{1}{2} \dot{\widetilde{\mathbf{M}}} \mathbf{e}_{v}+k_{i}\left(\int \mathbf{e}_{v} d t\right)\right] \\
& =\mathbf{e}_{v}^{T}\left[\frac{1}{2}(\dot{\widetilde{\mathbf{M}}}-2 \widetilde{\mathbf{C}})-\left(k_{p} \widetilde{\mathbf{M}}+k_{p} \mathbf{1}\right)\right] \mathbf{e}_{v}
\end{aligned}
$$

Now, recalling the skew-symmetry property of the matrix $\dot{\overrightarrow{\mathbf{M}}}-2 \widetilde{\mathbf{C}}$ in eq. (32), $\dot{V}$ can be written as

$$
\dot{V}=-\mathbf{e}_{v}^{T}\left(k_{p} \widetilde{\mathbf{M}}+k_{p} \mathbf{1}\right) \mathbf{e}_{v}
$$

which is negative-definite, since $\widetilde{\mathbf{M}}$ and $k_{p} \mathbf{1}$ are both positive-definite. This implies that $\mathbf{e}_{v}$ converges asymptotically to zero, thereby proving that the devised control law is capable of stabilizing the nonlinear time-varying error system (43).

Once we obtain $\tau$, the tractive forces of the two tracks are known. Further, the track slips $\sigma$ for both tracks can be calculated as per eq. (23). The input 
angular velocities of the two track drive sprockets can thus be obtained from eqs. (2a) and (2b), respectively.

The complete control scheme of the trajectory tracking problem for tracked vehicles is illustrated in Fig. 4 ,

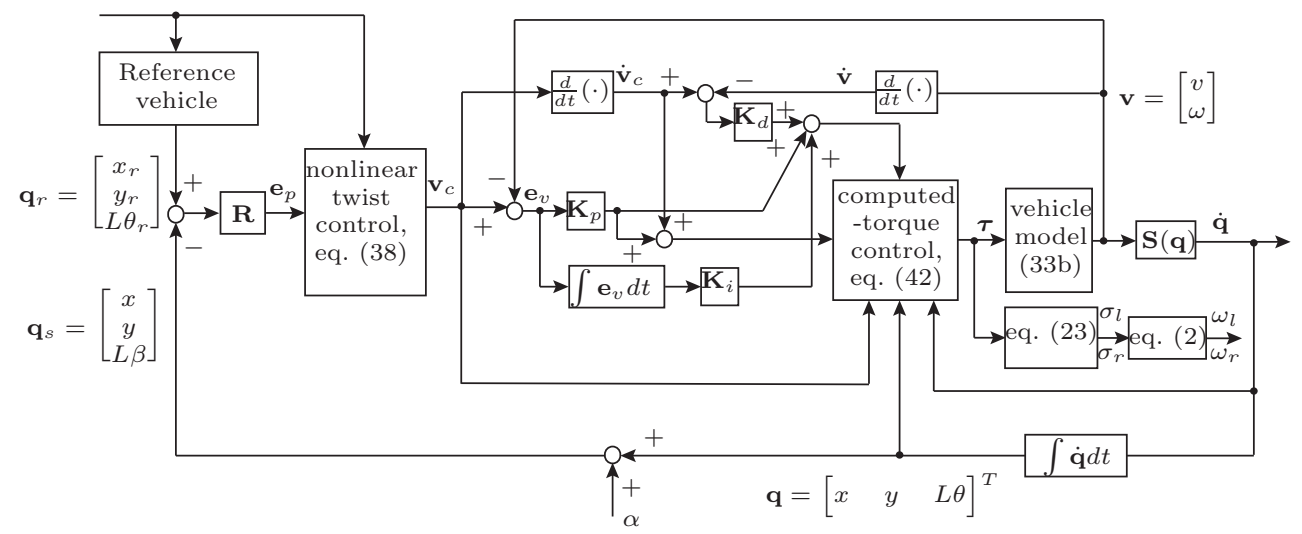

Figure 4: Control scheme of the trajectory-following of the tracked vehicle

\section{Simulation Results}

The proposed control strategy is implemented using a numerical example. The tracked vehicle is to follow a desired circular trajectory of $10 \mathrm{~m}$ radius, at a constant angular velocity of $0.8 \mathrm{rad} / \mathrm{s}$, and an initial pose $[-1,-2,0]^{T}$. The vehicle is assumed to maneuver on hard, horizontal ground, with the terrain and vehicle design parameters listed in Table 1

The reference circular trajectory is given in eq. (35). with the referencevelocity and angular-velocity values $v_{r}=8 \mathrm{~m} / \mathrm{s}$ and $\omega_{r}=-0.8 \mathrm{rad} / \mathrm{s}$, respectively.

The overall simulation time is $10 \mathrm{~s}$, with a time step of $0.001 \mathrm{~s}$. The initial angular velocity of the vehicle is given as $\omega_{0}=0$.

The control gains are tuned during different simulation tests, with the final values listed below:

$$
k_{x}=12, \quad k_{y}=6.3, \quad k_{\theta}=3.3, \quad k_{p}=36, \quad k_{i}=30, \quad k_{d}=16
$$


Table 1: Design parameters for simulation and test data Vehicle Design Parameters

\begin{tabular}{l|l}
\hline Mass of vehicle $m(\mathrm{~kg})$ & 3700 \\
Sprocket radius $r(\mathrm{~m})$ & 0.50 \\
Track contact length $l(\mathrm{~m})$ & 5.0 \\
Track width $h(\mathrm{~m})$ & 0.30 \\
Tread of vehicle $b(\mathrm{~m}) \quad 1.1$ \\
\hline \multicolumn{2}{c}{ Terrain Parameters } \\
\hline Soil shear deformation modulus $K(\mathrm{~m})$ & 0.02 \\
Friction coefficient between tracks and terrain $\mu$ & 0.9 \\
Apparent cohesion $c(\mathrm{~Pa})$ & 70000 \\
Angle of internal shearing $\phi(\mathrm{rad})$ & 0.67 \\
motion resistance coefficient in the longitudinal direction $\mu_{l}$ & 0.6 \\
motion resistance coefficient in the lateral direction $\mu_{t}$ & 0.8 \\
\hline
\end{tabular}

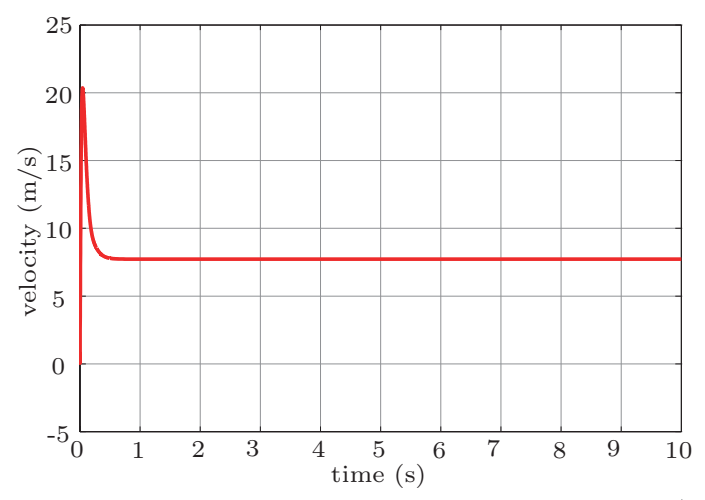

Figure 5: The velocity $v$ of the vehicle

Figures 5 and 6 illustrate the time-history of the velocity and the angular velocity of the tracked vehicle, respectively. It is apparent that the velocity does not converge from the initial time to $0.33 \mathrm{~s}$, which denotes unstable motion before $0.33 \mathrm{~s}$, while motion is stabilized for the balance of the simulation time.

The pose time-histories of the tracked vehicle in the plane, i.e., the planar position and orientation, are plotted in Fig. 7 .

Figure 8 shows the path followed by the tracked vehicle, represented by the red triangle, along a desired circular path on the horizontal plane. It is observed that, due to the significant slip at the initial stage of motion, the trajectory of the tracked vehicle does not converge to the desired path; however, the vehicle 


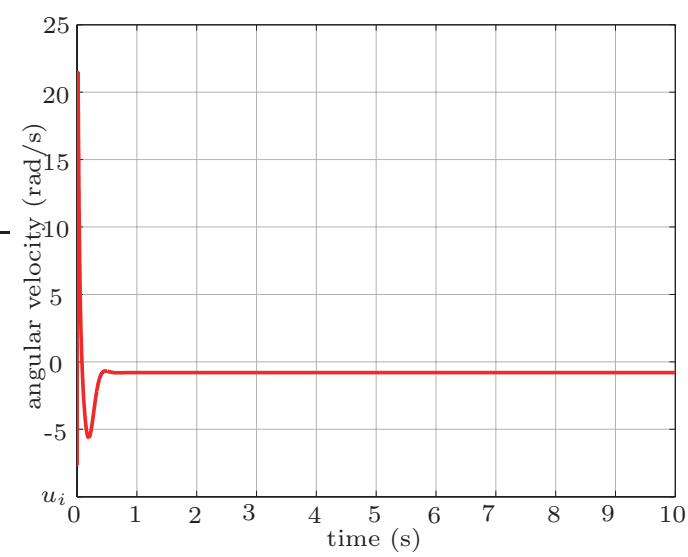

Figure 6: The angular velocity $\omega$ of the vehicle

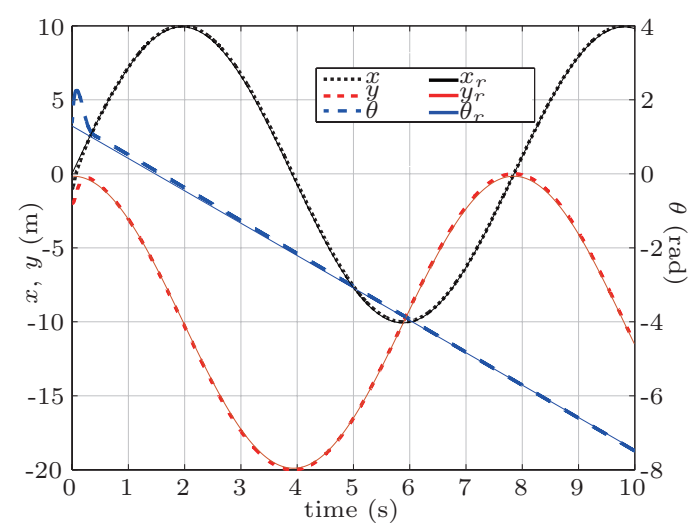

Figure 7: Pose of the vehicle

can follow the desired path quite closely after a significantly short period.

The angular velocity and slip ratio of the left and the right tracks are plotted in Figs. 9 and 10, respectively. Consistent with the trajectory of the vehicle, at the initial stage of motion, both the angular velocity and slip ratio do not converge, but they do reach stable values after a short lapse of time.

\section{Conclusions}

The authors proposed a motion control methodology, using the back-stepping method, based on a modified Proportional-Integral-Derivative (PID) computed- 


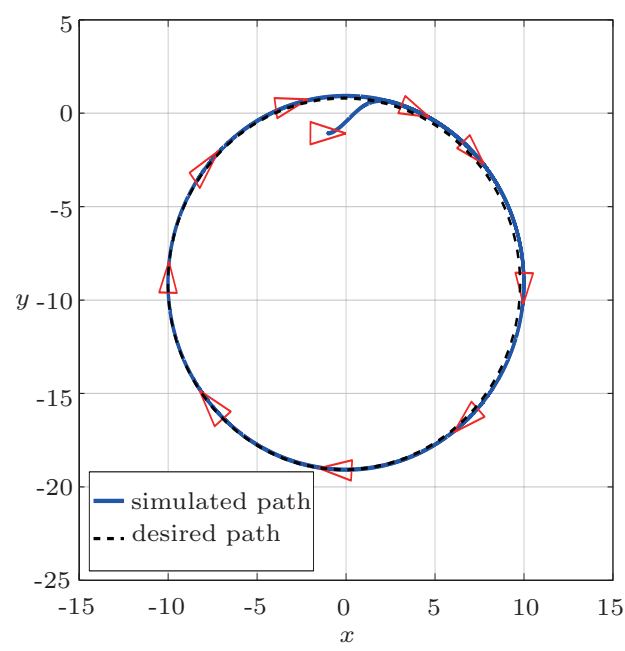

Figure 8: Vehicle following a desired circular path

torque controller. The pose and twist of the tracked vehicle platform, including the effects of slippage, are estimated using a biaxial accelerometer strapdown. Simulation examples are used to verify the proposed approach. In order to capture the nonlinear track-terrain interactions on horizontal, hard terrain, the governing equations of the system are derived, including slippage during skidsteering. The mathematical model thus formulated is mainly used for tracked mining blasthole drilling rigs, which normally maneuver on hard, horizontal ground. Therefore, the model assumes that the tractive shear stress is distributed evenly on both tracks. However, the minor shift effect in the center of rotation along the longitudinal direction due to unevenly distributed shear stress, in practice, can still be corrected by the trajectory-tracking controller designed in this paper. The proposed controller is a straightforward approach and can be readily applied in practical applications. The simulation results illustrate the high accuracy of the motion-control performance. The asymptotic stability of the system is guaranteed by Lyapunov theory. 


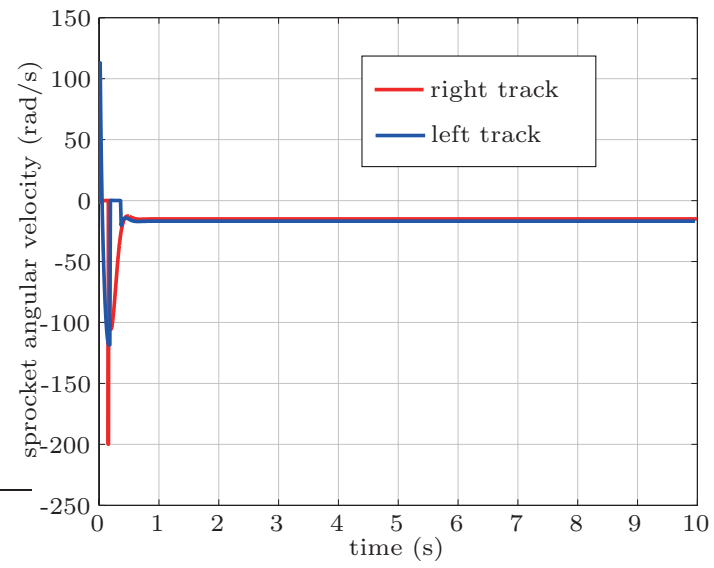

Figure 9: Angular velocities of the sprockets of the left and right tracks

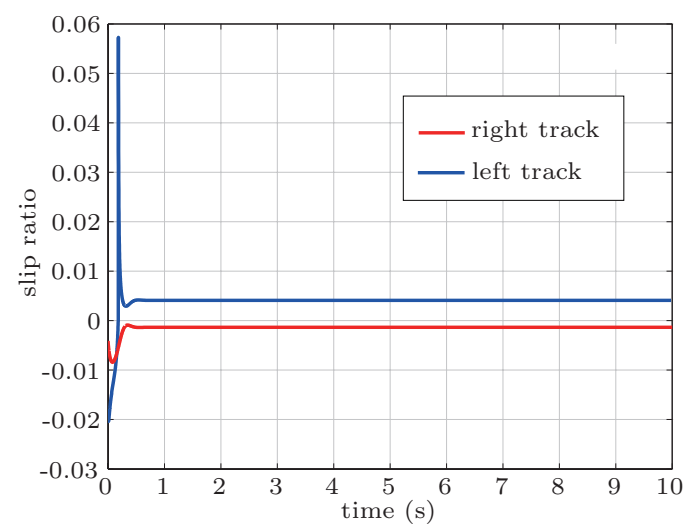

Figure 10: Slip ratio of the left and right tracks

\section{Acknowledgements}

The support from the Fonds québecois de la recherche sur la nature et les technologies (FQRNT) (Grant No. 241261) and the Natural Sciences and Engineering Research Council of Canada (NSERC) through a Discovery Grant (No. 223213) is dutifully acknowledged.

\section{References}

[1] P. Elinas, S. Singh, Drill hole planning, US Patent App. 13/264,740, 2012. 
[2] P. Darling, SME Mining Engineering Handbook (3rd Edition), Society for Mining, Metallurgy, and Exploration (SME), 2011.

[3] P. Corke, J. Roberts, J. Cunningham, D. Hainsworth, Mining robotics, in: B. Siciliano, O. Khatib (Eds.), Springer Handbook of Robotics, Springer, Berlin, Heidelberg, 2008, Ch. 49, pp. 1127-1150.

[4] J. Y. Wong, Theory of Ground Vehicles, Fourth Edition, John Wiley \& Sons, 2008.

[5] M. Ahmad, V. Polotski, R. Hurteau, Path tracking control of tracked vehicles, in: Proceedings of the 2000 IEEE International Conference on Robotics \& Automation, Vol. 3, San Francisco, CA, 2000, pp. 2938-2943.

[6] B. Zhou, Y. Peng, J. Han, UKF based estimation and tracking control of nonholonomic mobile robots with slipping, in: 2007 IEEE International Conference on Robotics and Biomimetics (ROBIO), 2007, pp. 2058-2063.

[7] R. Hennessy, F. Oppolzer, X. Fan, S. Singh, H. Durrant-Whyte, System and method for autonomous navigation of a tracked or skid-steer vehicle, US Patent 8,612,084, 2013.

[8] J.-P. P. Laumond, Robot Motion Planning and Control, Springer-Verlag New York, Inc., Secaucus, NJ, USA, 1998.

[9] R. W. Brockett, Asymptotic stability and feedback stabilization, in: Differential Geometric Control Theory, Birkhauser, 1983, pp. 181-191.

[10] A. M. Bloch, M. Reyhanoglu, N. H. McClamroch, Control and stabilization of nonholonomic dynamic systems, IEEE Transactions on Automatic Control 37 (11) (1992) 1746-1757.

[11] I. Kolmanovsky, N. H. McClamroch, Developments in nonholonomic control problems, IEEE Control Systems 15 (6) (1995) 20-36. 
[12] M. Burke, Path-following control of a velocity constrained tracked vehicle incorporating adaptive slip estimation, in: 2012 IEEE International Conference on Robotics and Automation, 2012, pp. 97-102.

[13] Y. Kanayama, Y. Kimura, F. Miyazaki, T. Noguchi, A stable tracking control method for a non-holonomic mobile robot, in: Proc. IEEE/RSJ Int. Workshop on Intelligent Robots and Systems, Vol. 3, 1991, pp. 12361241.

[14] C. Samson, Time-varying feedback stabilization of car-like wheeled mobile robots, Int. J. Rob. Res. 12 (1) (1993) 55-64.

[15] D. Endo, Y. Okada, K. Nagatani, K. Yoshida, Path following control for tracked vehicles based on slip-compensating odometry, in: 2007 IEEE/RSJ International Conference on Intelligent Robots and Systems, 2007, pp. 2871-2876.

[16] R. Fierro, F. L. Lewis, Control of a nonholonomic mobile robot using neural networks, IEEE Transactions on Neural Networks 9 (4) (1998) 589-600.

[17] J.-M. Yang, J.-H. Kim, Sliding mode motion control of nonholonomic mobile robots, IEEE Control Systems 19 (2) (1999) 15-23.

[18] Z. Song, D. Zhao, J. Yi, X. Li, Robust motion control for nonholonomic constrained mechanical systems: sliding mode approach, in: Proceedings of the 2005, American Control Conference, Vol. 4, 2005, pp. 2883-2888.

[19] Y.-C. Chang, B.-S. Chen, Robust tracking designs for both holonomic and nonholonomic constrained mechanical systems: adaptive fuzzy approach, IEEE Transactions on Fuzzy Systems 8 (1) (2000) 46-66.

[20] T. Fukao, H. Nakagawa, N. Adachi, Adaptive tracking control of a nonholonomic mobile robot, IEEE Transactions on Robotics and Automation 16 (5) (2000) 609-615. 
[21] M.-S. Kim, J.-H. Shin, S.-G. Hong, J.-J. Lee, Designing a robust adaptive dynamic controller for nonholonomic mobile robots under modeling uncertainty and disturbances, Mechatronics 13 (5) (2003) 507 - 519.

[22] L. Xin, Q. Wang, J. She, Y. Li, Robust adaptive tracking control of wheeled mobile robot, Robotics and Autonomous Systems 78 (2016) 36-48.

[23] T. T. Hoang, D. T. Hiep, B. G. Duong, T. Q. Vinh, Trajectory tracking control of the nonholonomic mobile robot using torque method and neural network, in: IEEE 8th Conference on Industrial Electronics and Applications (ICIEA), 2013, pp. 1898-1803.

[24] G. Klančar, I. S̆krjanc, Tracking-error model-based predictive control for mobile robots in real time, Robotics and Autonomous Systems 55 (6) (2007) 460-469.

[25] R. Fierro, F. L. Lewis, Control of a nonholonomic mobile robot: backstepping kinematics into dynamics, in: Proceedings of the 34th IEEE Conference on Decision and Control, Vol. 4, New Orleans, LA, December 1995, pp. 3805-3810.

[26] Y. Choi, W. Chung, PID Trajectory Tracking Control for Mechanical Systems, Lecture Notes in Control and Information Sciences, Springer Berlin Heidelberg, 2004.

[27] T. I. Fossen, O.-E. Fjellstad, Nonlinear modelling of marine vehicles in 6 degrees of freedom, Mathematical Modelling of Systems 1 (1) (1995) 17-27.

[28] M. W. Spong, S. Hutchinson, M. Vidyasagar, Robot Modeling and Control, John Wiley \& Sons, Inc., Hoboken, NJ, 2006.

[29] P. J. From, I. Schjølberg, J. T. Gravdahl, K. Y. Pettersen, T. I. Fossen, On the boundedness and skew-symmetric properties of the inertia and Coriolis matrices for vehicle-manipulator systems, IFAC Proceedings Volumes 43 (16) (2010) 193-198. 
[30] V. Polotski, Enhanced path planning for articulated and skid steering mining vehicles, in: 15th Triennial World Congress of the International Federation of Automatic Control (IFAC), Barcelona, Spain, July 2002.

[31] S. Hong, J.-S. Choi, H.-W. Kim, M.-C. Won, S.-C. Shin, J.-S. Rhee, H.-u. Park, A path tracking control algorithm for underwater mining vehicles, Journal of Mechanical Science and Technology 23 (8) (2009) 2030-2037.

[32] J. Angeles, The role of the rotation matrix in the teaching of planar kinematics, Mechanism and Machine Theory 89 (2015) 28-37.

[33] Z. Shiller, W. Serate, Trajectory planning of tracked vehicles, J. Dyn. Sys., Meas., Control 117 (4) (1995) 619-624.

[34] S. Ostrovskaya, J. Angeles, Nonholonomic systems revisited within the framework of analytical mechanics, Applied Mechanics Reviews 51 (7) (1998) 415-433.

[35] J. I. Neimark, N. A. Fufaev, Dynamics of Nonholonomic Systems, Vol. 33 of Translations of Mathematical Monographs, Amer. Mathem. Society, Providence, 1972 .

[36] M. G. Bekker, Theory of Land Locomotion: the Mechanics of Vehicle Mobility, The University of Michigan Press, 1962.

[37] J. Peck, P. Hendrics, GPS-based navigation systems on mobile mining equipment for open-pit mine, CIM Bulletin, Barcelona, Spain, 1997.

[38] A. Caspani, C. Comi, A. Corigliano, G. Langfelder, A. Tocchio, Compact biaxial micromachined resonant accelerometer, Journal of Micromechanics and Microengineering 23 (10) (2013) 105012-1-10.

[39] T. Zou, J. Angeles, An algorithm for rigid-body angular velocity and attitude estimation based on isotropic accelerometer strapdowns, ASME Journal of Applied Mechanics 85 (6) (2018) 061010-1-10. 
[40] Y. Kanayama, Y. Kimura, F. Miyazaki, T. Noguchi, A stable tracking control method for an autonomous mobile robot, in: Proceedings., IEEE International Conference on Robotics and Automation, 1990, pp. 384-389 vol.1.

[41] Y. Kanayama, A. Nilipour, C. A. Lelm, A locomotion control method for autonomous vehicles, Vol. 2, IEEE International Conference on Robotics and Automation, Philadelphia, PA, 1988, pp. 1315-1317.

[42] W. L. Nelson, I. J. Cox, Local path control for an autonomous vehicle, Vol. 3, IEEE International Conference on Robotics and Automation, Philadelphia, PA, 1988, pp. 1504-1510. 